\title{
Evolution, expansion and expression of the Kunitz/BPTI gene family associated with long- term blood feeding in Ixodes Scapularis
}

Shao-Xing Dai ${ }^{1,2}$, Ai-Di Zhang ${ }^{1,2}$ and Jing-Fei Huang ${ }^{1,2^{*}}$

\begin{abstract}
Background: Recent studies of the tick saliva transcriptome have revealed the profound role of salivary proteins in blood feeding. Kunitz/BPTI proteins are abundant in the salivary glands of ticks and perform multiple functions in blood feeding, such as inhibiting blood coagulation, regulating host blood supply and disrupting host angiogenesis. However, Kunitz/BPTI proteins in soft and hard ticks have different functions and molecular mechanisms. How these differences emerged and whether they are associated with the evolution of long-term blood feeding in hard ticks remain unknown.

Results: In this study, the evolution, expansion and expression of Kunitz/BPTI family in Ixodes scapularis were investigated. Single- and multi-domain Kunitz/BPTI proteins have similar gene structures. Single-domain proteins were classified into three groups (groups I, II and III) based on their cysteine patterns. Group I represents the ancestral branch of the Kunitz/BPTI family, and members of this group function as serine protease inhibitors. The group I domain was used as a module to create multi-domain proteins in hard ticks after the split between hard and soft ticks. However, groups II and III, which evolved from group I, are only present and expanded in the genus Ixodes. These lineage-specific expanded genes exhibit significantly higher expression during long-term blood feeding in Ixodes scapularis. Interestingly, functional site analysis suggested that group II proteins lost the ability to inhibit serine proteases and evolved a new function of modulating ion channels. Finally, evolutionary analyses revealed that the expansion and diversification of the Kunitz/BPTI family in the genus /xodes were driven by positive selection.

Conclusions: These results suggest that the differences in the Kunitz/BPTI family between soft and hard ticks may be linked to the evolution of long-term blood feeding in hard ticks. In Ixodes, the lineage-specific expanded genes (Group II and III) lost the ancient function of inhibiting serine proteases and evolved new functions to adapt to long-term blood feeding. Therefore, these genes may play a profound role in the long-term blood feeding of hard ticks. Based our analysis, we propose that the six genes identified in our study may be candidate target genes for tick control.
\end{abstract}

\section{Background}

Ticks are classified into two major families: Ixodidae (hard ticks) and Argasidae (soft ticks) [1,2]. The family Ixodidae is further divided into two groups, Prostriata and Metastriata. Prostriata contains only a single genus, Ixodes. In contrast, Metastriata contains four subfamilies: Amblyomminae, Haemaphysalinae, Hyalomminae,

\footnotetext{
* Correspondence: huangjf@mail.kiz.ac.cn

'School of Life Sciences, University of Science and Technology of China, Hefei, Anhui 230027, P.R. China

Full list of author information is available at the end of the article
}

and Rhipicephalinae [1,2]. All ticks are external bloodfeeding parasites of mammals, birds and reptiles throughout the world $[3,4]$. They can transmit a wide variety of pathogens causing several human and animal diseases, including Lyme disease, human granulocytic anaplasmosis, and human babesiosis [5,6]. However, hard and soft ticks display different feeding strategies. Hard ticks feed on blood for a few days to over one week, whereas soft ticks typically feed on blood for minutes to hours [7]. The evolutionary drivers of long-term blood feeding in hard ticks remain unknown.
C Biomed Central

() 2012 Dai et al; licensee BioMed Central Ltd. This is an Open Access article distributed under the terms of the Creative Commons Attribution License (http://creativecommons.org/licenses/by/2.0), which permits unrestricted use, distribution, and reproduction in any medium, provided the original work is properly cited. 
Blood feeding is a complex process. When attempting to feed the blood from their hosts, ticks face the problem of host defenses, such as hemostasis, inflammation, and immunity [7-10]. Recent studies of the saliva transcriptome of ticks [11-20] and some review papers $[7,10,21]$ have demonstrated that tick salivary proteins play a profound role in the process of blood feeding. Kunitz/BPTI proteins are abundant in the salivary glands (SGs) of ticks [11-18], suggesting that they have important roles in blood feeding. The Kunitz/BPTI domain is an ancient and widespread domain with a disulfide-rich alpha + beta fold that is stabilized by three highly conserved disulfide bridges with the bonding patterns 1-6, 2-4, and 3-5 [22-24]. The typical Kunitz/BPTI domain has a cysteine pattern of $\mathrm{CX}(8) \mathrm{CX}(15) \mathrm{CX}(7) \mathrm{CX}$ (12)CX(3)C [22-24]. Ticks exhibit other cysteine patterns, such as $\mathrm{CX}(8) \mathrm{CX}(18) \mathrm{CX}(5) \mathrm{CX}(12) \mathrm{CX}(3) \mathrm{C}$ and $\mathrm{CX}$ $(5,6) \mathrm{CX}(15) \mathrm{CX}(8) \mathrm{CX}(11) \mathrm{CX}(3) \mathrm{C}$, in the Kunitz/BPTI proteins due to insertions and deletions (indels) $[12,15]$. Additionally, Kunitz/BPTI proteins in the SGs and midgut of ticks have signal peptides that allow them to be secreted into the extracellular medium [15,25]. Interestingly, the Kunitz/BPTI domain was used as a module to construct multi-domain Kunitz/BPTI proteins in ticks. Therefore, some tick proteins have complex domain architectures containing two or more Kunitz/BPTI domains $[12,15]$. The domain architectures and sequences of the Kunitz/BPTI proteins are highly divergent between soft and hard ticks [8,12,15]. Furthermore, the various Kunitz/BPTI proteins can perform different functions. In soft ticks, Kunitz/BPTI proteins function as anti-hemostatic factors by inhibiting blood coagulation and platelet aggregation $[7,8,26]$. In hard ticks, Kunitz/BPTI proteins can regulate host blood supply [24] and disrupt host angiogenesis and wound healing [27].

How the functional differences and complex domain architectures of Kunitz/BPTI proteins emerged and whether this evolution is associated with differences in blood-feeding strategies between soft and hard ticks remain unclear. Studying the evolution and expression of Kunitz/BPTI gene family between soft and hard ticks can help answer these questions. The evolution of the Kunitz/BPTI family in soft ticks has been previously studied $[11,26]$. These studies revealed that the blood coagulation and platelet aggregation inhibitors (Kunitz/ BPTI proteins) evolved in the ancestral soft tick lineage and persisted in all soft ticks. However, to our knowledge, there has not been a comprehensive and systematic study on the evolution and expression of Kunitz/ BPTI gene family in hard ticks. The hard tick Ixodes scapularis and its close relatives, Ixodes pacificus and Ixodes ricinus, are the most important ticks because they transmit the majority of emerging human disease pathogens $[28,29]$. The genome and saliva transcriptome of Ixodes scapularis are now available $[15,18,28]$, which provides an opportunity to study the evolution and expression of Kunitz/BPTI gene family in Ixodes scapularis.

In our study, we systematically examined the evolution, expansion and expression of Kunitz/BPTI gene family in Ixodes scapularis. We then compared the characteristics of this family between soft and hard ticks by sequence, structure and gene expression analyses. Based on these analyses, we illustrated the profound role of this family in long-term blood feeding and discuss the reasons for different blood-feeding strategies between hard and soft ticks. Finally, we proposed that the six genes (Table 1) with highly dynamic expression identified in our study might be candidate target genes for tick control.

\section{Results \\ Identification, classification and gene structure of the Kunitz/BPTI family in all ticks}

The sequence search strategy used here was described in Methods and additional file (Additional File 1). We retrieved 368 sequences in the NR database at the NCBI website. After removing redundant sequences (Additional File 2), partial sequences and other sequences without the cysteine motif of the Kunitz/BPTI domain, a total of 303 Kunitz/BPTI proteins were recognized in

Table 1 Six differentially expressed Kunitz/BPTI genes in Ixodes scapularis.

\begin{tabular}{llllll}
\hline Unigene & Protein & $\mathbf{R}$ & ISUF & IS6-12 & IS18-24 \\
\hline Isc.218 & AAY66618.1 (group II) & 241.40 & 0 & 17 & 259 \\
\hline Isc.190 & AAM93606.1(group II) & 19.74 & 0 & 0 \\
\hline Isc.255 & AAM93612.1(group II) & 10.96 & 0 & 0 & 0 \\
\hline Isc.196 & AAM93610.1(group II) & 10.96 & 0 & 0 & 0 \\
\hline Isc.180 & AAM93635.1(group III) & 23.03 & 0 & 0 \\
\hline Isc.179 & AAM93632.1(group III) & 9.87 & 0 & 0 & 0 \\
\hline
\end{tabular}

Note. This table lists the 6 genes with $\mathrm{R}>9$. The first column is the name of Unigene cluster. The second column is protein corresponding to Unigene cluster, with the classification in bracket. The third column gives the likelihood statistic R. The next four columns show the number of ESTs in each of the four libraries that belong to the Unigene cluster. The total numbers of EST sequences in each of the libraries are 1026, 952,1995 and 1993. 
ticks and used for analysis in this study. Among the 303 sequences, there are 162 single-domain Kunitz/BPTI proteins (One-KU), 92 two-domain Kunitz/BPTI proteins (Two-KU), 16 three-domain Kunitz/BPTI proteins (Three-KU), 9 four-domain Kunitz/BPTI proteins (Four$\mathrm{KU}), 17$ five-domain Kunitz/BPTI proteins (Five-KU), and 3 seven-domain Kunitz/BPTI proteins (Seven-KU). Six-KU proteins were not present. Signal peptides of the 303 sequences were predicted using the SignalP 4.0 server [30]. We found that most of the Kunitz/BPTI proteins (205/303 sequences) have signal peptides. Signal peptides were detected in each type of Kunitz/BPTI protein, indicating that Kunitz/BPTI proteins perform their function mainly in the extracellular medium.

The genome data of Ixodes scapularis contain rich information about the organization between Kunitz/ BPTI gene loci and their gene structures. By aligning mRNA to genomic sequences, we obtained gene structures and organization for the Kunitz/BPTI gene loci (Figure 1). Single-domain proteins have similar gene structure. Furthermore, single and multi-domain Kunitz/BPTI proteins have similar gene structure. The first exon encodes a signal peptide, and the Kunitz/BPTI domain is encoded in a single exon (Figure 1A). Multiple exons arrayed in tandem in the gene contribute to

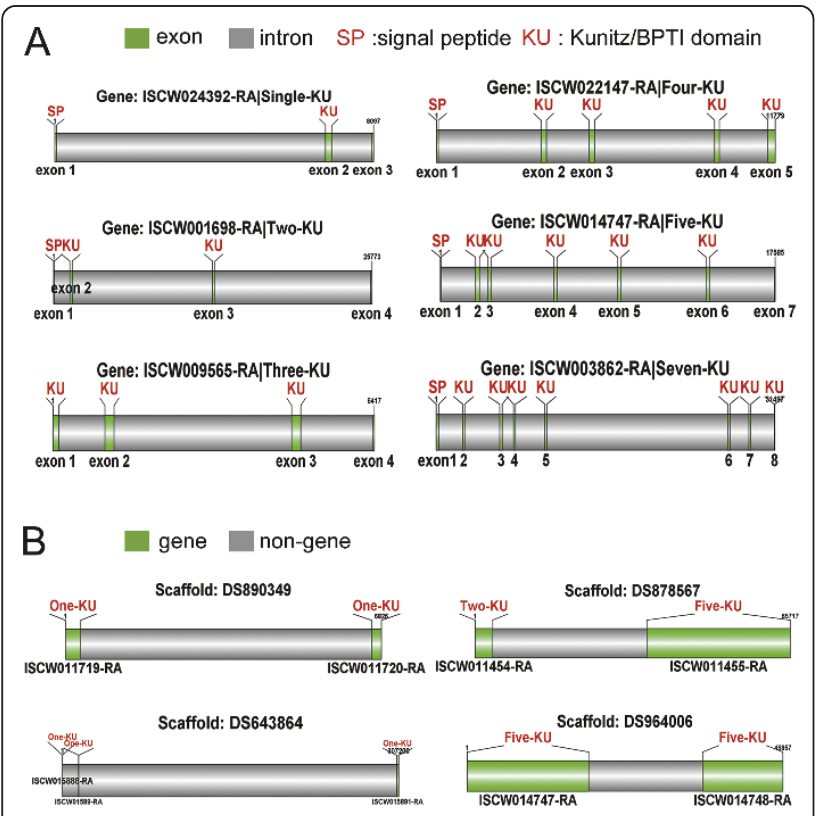

Figure 1 Gene structures of Kunitz/BPTI proteins and their distributions in the genome. (A), Gene structures of Kunitz/BPTI proteins. The gene structures of single and multi-domain Kunitz/ BPTI proteins show similar characters. Exons were colored with green and numbered below. SP: signal peptide; KU: Kunitz/BPTI domain; (B), the distributions of Kunitz/BPTI gene. Some Kunitz/BPTI genes are arranged in tandem in the genome. Genes were colored with green. multi-domain Kunitz/BPTI proteins. The length of the introns is divergent. However, the Kunitz/BPTI domainencoding exon has an average length of $180 \mathrm{bp}$, which encodes the 60-aa domain. Some Kunitz/BPTI genes (9/ $55)$ are arranged in tandem in the genome (Figure 1B), while other Kunitz/BPTI genes (46/55) are dispersed throughout the genome.

Additionally, we obtained functional information for the Kunitz/BPTI proteins [20,25,31-34]. All 303 sequences are listed with related information, such as the species name, gene name, domain architecture, length, signal peptide and function (Additional File 3).

\section{Evolution of single-domain Kunitz/BPTI proteins: Evidence} for lineage-specific expansion in Ixodes ticks

To clarify the phylogenetic relationships of singledomain Kunitz/BPTI proteins in Ixodes scapularis and compare them with other tick Kunitz/BPTI proteins, we first analyzed the Kunitz/BPTI proteins in Ixodes scapularis. Then, all tick Kunitz/BPTI proteins were gathered for further analysis. A total of 80 Kunitz/BPTI proteins in Ixodes scapularis are clustered into three clades (named groups I, II and III) (Figure 2A and Additional File 4). Groups II and III are highly supported with posterior probabilities of 0.99 and 1 in Bayesian inference (MB) tree and bootstrap values of $61 \%$ and $100 \%$ (NJ tree) and $62 \%$ and $100 \%$ (ML tree), respectively. In contrast, group I forms one clade with lower support values of 0.79 in the MB tree and 0.75 in the NJ tree. The proteins group I do not cluster into one clade in the ML tree (Additional File 4). The Low supports for the three groups may be due to the long branches and divergence in the group. Groups I, II and III follow the cysteine patterns CX(8)CX(15)CX(7)CX(12)CX(3)C, CX(8)CX (18)CX(5)CX(12)CX(3)C and CX(5,6) CX(15)CX(8)CX (11) $\mathrm{CX}(3) \mathrm{C}$, respectively, although there are exceptions (Figure 2B and Additional File 5). The pattern of group I is the typical cysteine pattern of the Kunitz/BPTI family, and this motif is also common in Bilateria and Cnidaria [24,35-39]. This suggests that group I represents the ancestral branch of the Kunitz/BPTI family in Ixodes scapularis.

Next, we extended our analysis of single-domain Kunitz/BPTI proteins from Ixodes scapularis to all ticks. Group I proteins in Ixodes scapularis are distributed widely, and they do not form a monophyletic clade (Figure 3 and Additional File 6), which further indicates that group I represents the ancestral branch of the Kunitz/BPTI family in Ixodes scapularis. By contrast, groups II and III form two separate monophyletic clades with high support (posterior probabilities of 0.92 and 0.97 in the MB tree and bootstrap values of $70 \%$ and $97 \%$ in the NJ tree and $70 \%$ and $84 \%$ in the ML tree, respectively) (Figure 3 and Additional File 6). This 


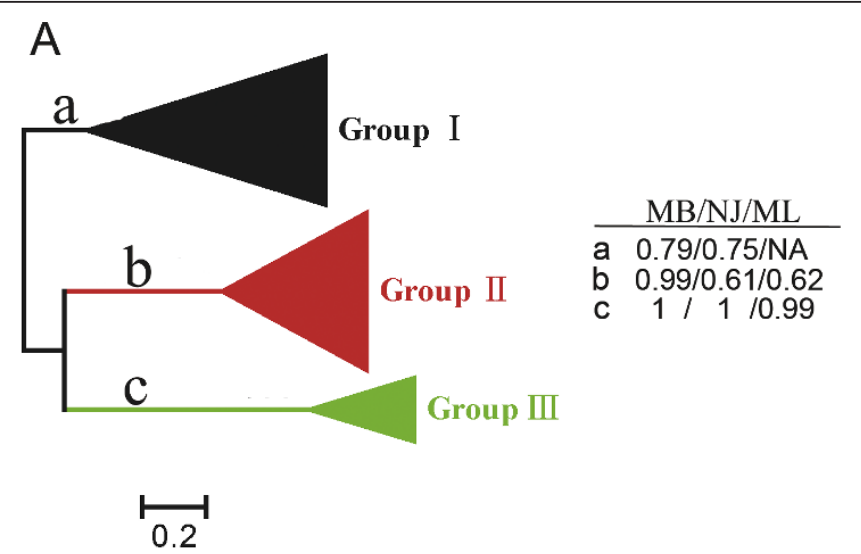

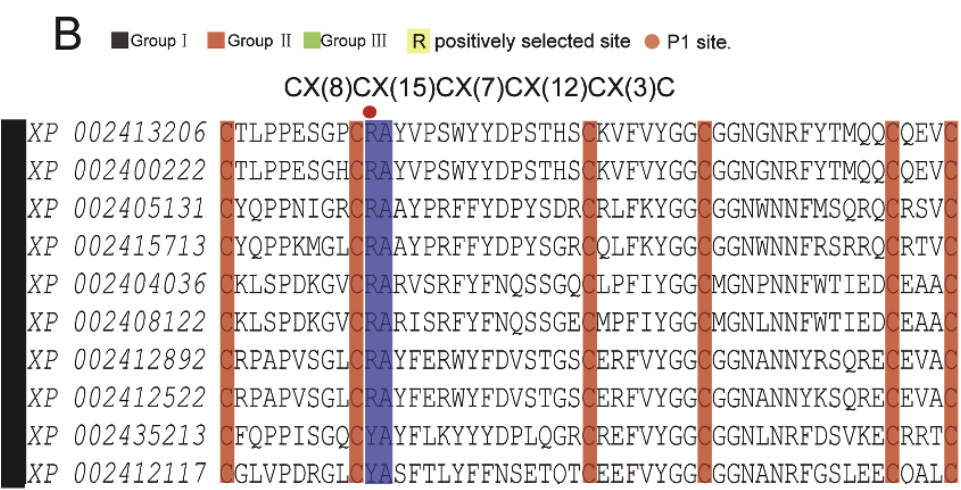

AAY 66687

AAY 66615

AAY 66656

AAY 66618

AAY 66764

AAM93607

AAY 66511

AAM93612

AAM93613

$\mathrm{CX}(8) \mathrm{CX}(18) \mathrm{CX}(5) \mathrm{CX}(12) \mathrm{CX}(3) \mathrm{C}$

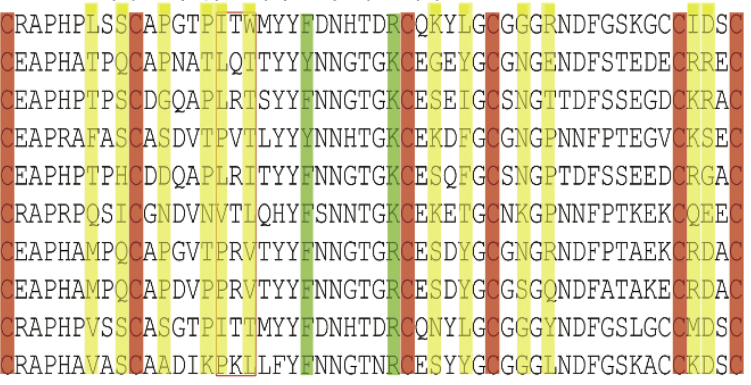

CX(5)CX(15)CX(8)CX(11)CX(3)C

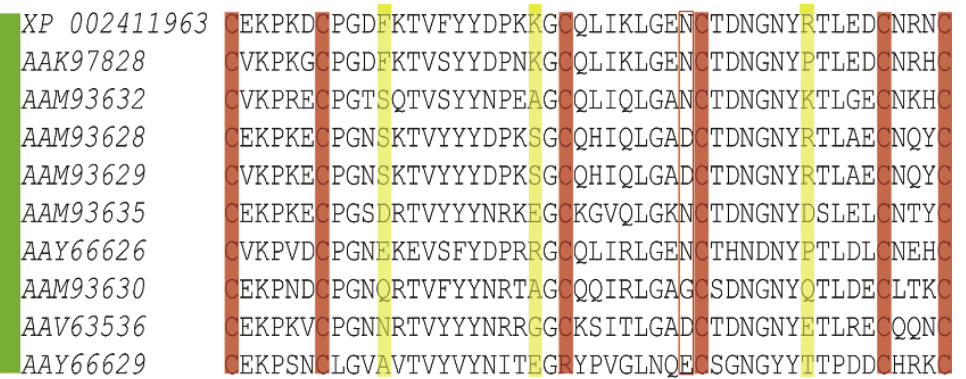

Figure 2 Cluster and alignment of single-domain Kunitz/BPTI proteins in Ixodes scapularis. (A), MB tree for single-domain Kunitz/BPTI proteins in Ixodes scapularis. The length of each branch is proportional to the estimated number of substitutions. Bar denotes $20 \%$ substitutions per site. The support values for each group (in $M B, N J$, and $M L$ tree) were shown in right panel. "NA" mans that proteins in group1 do not cluster into one clade (the bootstrap values $<50 \%$ ) in the ML tree of single-domain Kunitz/BPTI proteins in Ixodes scapularis. (Note: the value $50 \%$ is cutoff value in the Tree Finder.) (B), Alignment of representing single-domain Kunitz/BPTI proteins in Ixodes scapularis. The alignment only shows the part between the first and sixth cysteine (C1-C6) in Kunitz/BPTI protein. Red boxes indicate different insertions relative to group I. The six conserved cysteines were colored by red. Red circle indicates the position of $\mathrm{P}_{1}$ site. Key residues for serine proteases inhibition and ion channels modulating were highlighted in blue and green, respectively. Positively selected sites in group II and III were colored by yellow. 


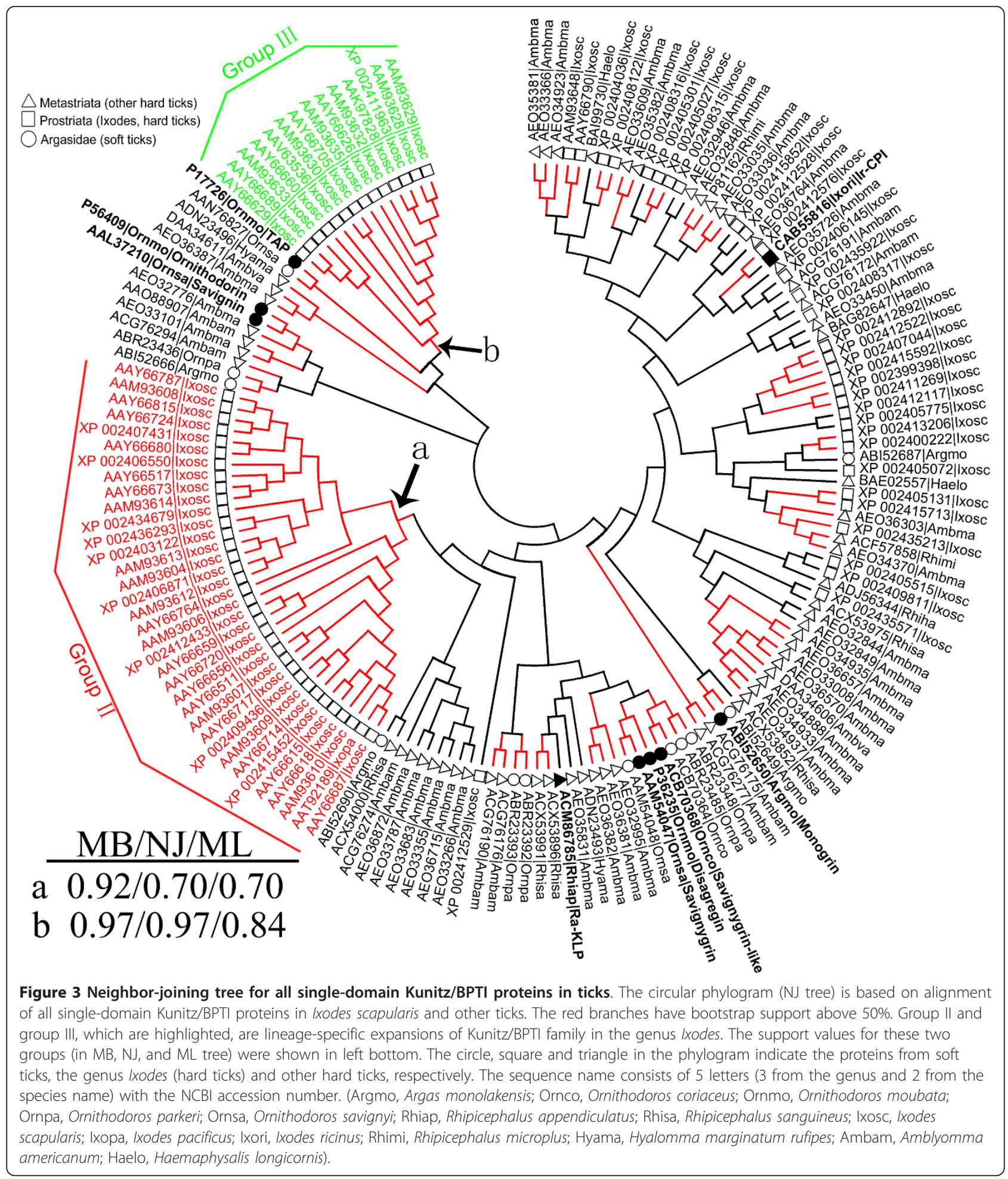

suggests that group II and III proteins may be only present in the genus Ixodes.

To confirm this argument, we searched the EST database of NCBI by TBLASTN. We were able to obtain some Kunitz/BPTI ESTs in Ixodes ricinus with translated protein sequences containing cysteine patterns from groups II and III (Additional File 7). However, this cysteine pattern was not found in other tick genera ESTs. Furthermore, we searched the genome of an additional hard tick (Rhipicephalus microplus) using 
TBLASTN but did not find any group II or III proteins. Taken together, we infer that the genes in groups II and III are only present in species of the genus Ixodes. Groups II and III are lineage-specific expansions of the Kunitz/BPTI family in Ixodes.

\section{Evolution of multi-domain Kunitz/BPTI proteins: The group I domain is a module for constructing multi- domain Kunitz/BPTI proteins}

There are many multi-domain Kunitz/BPTI proteins in ticks. Two-, three-, four- and five-KU proteins from all available tick sequences were aligned, and their corresponding phylogenetic trees were constructed. A phylogenetic tree for seven-KU proteins was not constructed because only three seven-KU proteins were found (one in Ixodes scapularis and two in Amblyomma maculatum). By carefully checking these phylogenetic trees, we found that two-KU proteins are widespread in both soft and hard ticks (Figure 4A). Intriguingly, the proteins that have more than two Kunitz/BPTI domains (three-, four-, five- and seven-KU) are only present in hard ticks (Figure 4B, C, and 4D). This indicates that many multi-
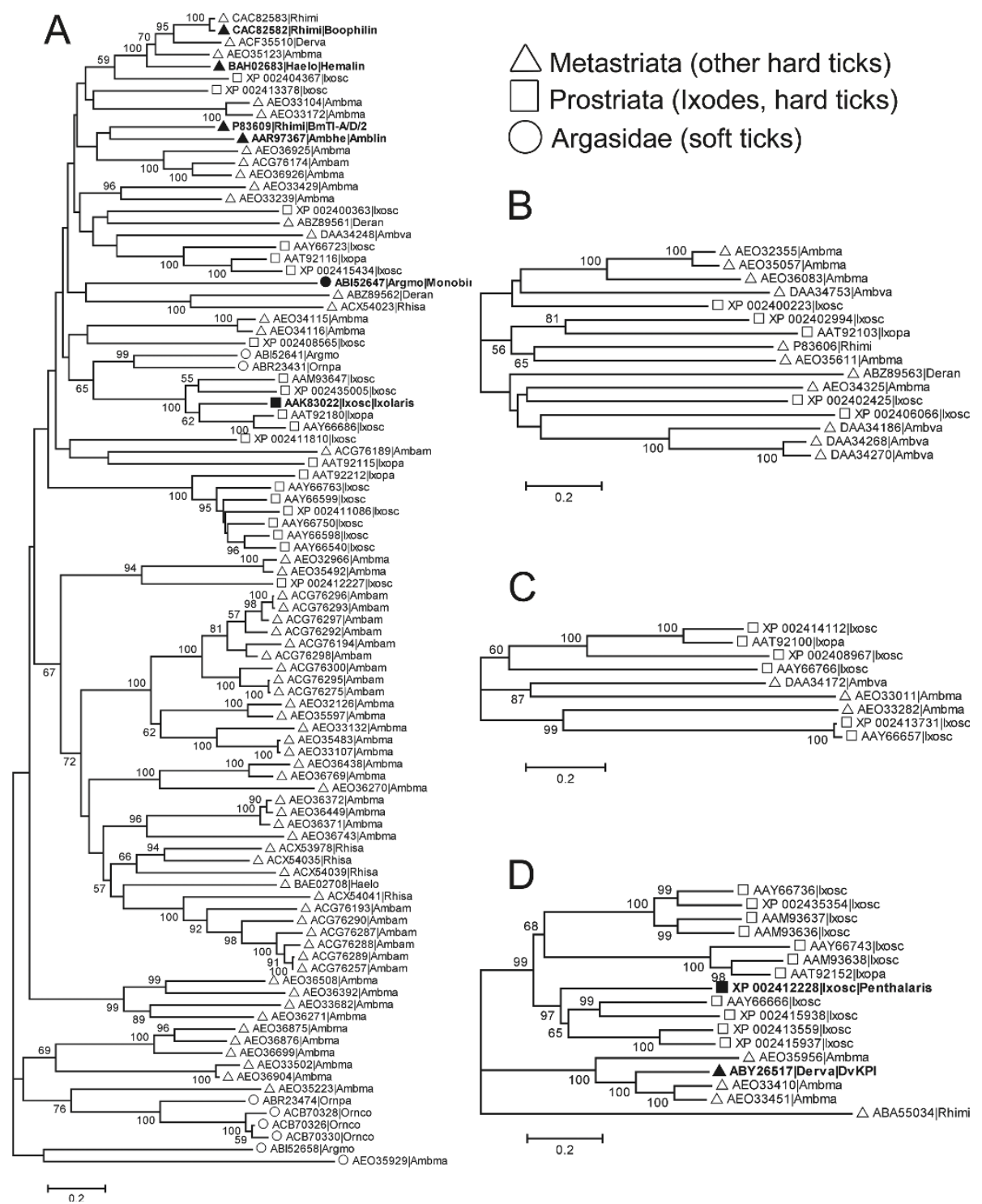

Figure 4 Neighbor-joining trees for multi-domain Kunitz/BPTI proteins in ticks. Neighbor-joining trees for Two-KU (A), Three-KU (B), Four$\mathrm{KU}$ (C) and Five-KU (D) proteins in ticks were reconstructed. The length of each branch is proportional to the estimated number of substitutions. Bar denotes $20 \%$ substitutions per site. The bootstrap values (> 50\%) were shown in branches. The circle, square and triangle in the phylogram indicate the proteins from soft ticks, the genus /xodes (hard ticks) and other hard ticks, respectively. The sequence name consists of 5 letters ( 3 from the genus and 2 from the species name) with the NCBI accession number. 
domain Kunitz/BPTI proteins were created in hard ticks during the evolution of the Kunitz/BPTI family. These multi-domain Kunitz/BPTI proteins may contribute to the different blood-feeding strategies of soft and hard ticks.

In our analysis, multiple Kunitz/BPTI domains in tandem contribute to multi-domain Kunitz/BPTI proteins. Because single-domain Kunitz/BPTI proteins were classified into three groups, we examined whether multidomain Kunitz/BPTI proteins arose from a single Kunitz/BPTI group or whether they originated from different Kunitz/BPTI groups. First, all multi-domain Kunitz/BPTI proteins in Ixodes scapularis were split into single-domain segments. Then, these single-domain segments were aligned together with all of the singledomain Kunitz/BPTI proteins in Ixodes scapularis, and the corresponding phylogenetic trees were constructed (Additional File 8). Again, groups II and III form two separate monophyletic clades with high support (posterior probabilities of 0.96 and 1 in the MB tree and bootstrap values of $77 \%$ and $96 \%$ in the NJ tree and $82 \%$ and $91 \%$ in the ML tree, respectively). In contrast, group I proteins are distributed widely, and they cluster with other domains from the different multi-domain Kunitz/ BPTI proteins. This indicates that only the group I domain was used as a module for constructing multidomain Kunitz/BPTI proteins, and group II and III proteins were not involved in the formation of multidomain Kunitz/BPTI proteins.

This hypothesis is also supported by the species distribution of the Kunitz/BPTI proteins. Groups II and III are only present in the genus Ixodes. However, multidomain Kunitz/BPTI proteins are widely present in hard ticks (including the genera Ixodes, Amblyomma, and Rhipicephalus) and soft ticks (in the case of two-KU proteins). This suggests that multi-domain Kunitz/BPTI proteins emerged earlier than the development of group II and III proteins. Therefore, it is impossible for multidomain Kunitz/BPTI proteins to have originated from group II and III domains.

\section{Functional site analysis of the Kunitz/BPTI family in Ixodes scapularis}

The typical Kunitz/BPTI protein has a conserved tertiary structure stabilized by three highly conserved disulfide bridges with the bonding patterns 1-6, 2-4, and 3-5 (Figure 5A). The structure-function relationship of Kunitz/BPTI proteins has been studied in prior studies [22,24,34,36,37,40-43]. Kunitz/BPTI proteins have two functions. One commonly accepted function is the inhibition of serine proteases $[8,44]$. The second function, which is relatively rare, is the blocking of ion channels $[24,45]$. At the "apex" of the inhibitor structure, a protruding loop (L1) penetrates deeply in the catalytic-site cleft of a protease to inhibit the protease via the $\mathrm{P}_{1}$ residue side chain (Lys15 in BPTI) $[24,36,46,47]$ (Figure 5A). In ion channel blockers, the "base" of the structure, which contains a $3^{10}$ helix and a $\beta$-turn, binds near the entrance of the pore of an ion channel to block the ion channel activity $[39,48-50]$ (Figure 5A).

The key residues for inhibiting serine proteases are the amino acids surrounding the $\mathrm{P}_{1}$ residue that are in direct contact with the protease [51]. Trypsin inhibitors have a basic residue ( $\mathrm{R}$ or $\mathrm{K}$ ) in the $\mathrm{P}_{1}$ position followed by a small residue (motif: $C_{2}[K / R] s$ ). Chymotrypsin inhibitors have a residue with a large side-chain $(\mathrm{F}, \mathrm{L}$, $N, Y$ ) in the $P_{1}$ position followed by a small residue (motif: $\mathrm{C}_{2}[\mathrm{~B}] \mathrm{s}$ ) (where $\mathrm{C}_{2}$, $\mathrm{B}$ and $\mathrm{s}$ indicate the second residue cysteine, a residue with a large side-chain and a small residue, respectively) [36]. Most of the proteins in group I exhibit the motifs $\mathrm{C}_{2}[\mathrm{~K} / \mathrm{R}] \mathrm{s}$ and $\mathrm{C}_{2}[\mathrm{~B}] \mathrm{s}$ (Figure $2 \mathrm{~B}$ and Additional File 5), which suggests that they are serine protease inhibitors. Therefore, group I proteins may function as anti-hemostatic factors by inhibiting serine proteases in the coagulation system. For example, the protein Ir-CPI (Figure 3) from Ixodes ricinus has the motif $\mathrm{C} 2[\mathrm{~K} / \mathrm{R}] \mathrm{s}$, and it can inhibit FXIIa, FXIa, and kallikrein [52].

However, proteins in groups II and III lack the key residues for inhibiting serine proteases (Figure 2B), suggesting that they lost this function. To investigate whether group II and III proteins have evolved new functions, we performed structure-based phylogenetic analysis (Figure 5B, C). Group III proteins form a monophyletic clade that does not include homologous structures (Figure $5 \mathrm{C}$ ), preventing us from inferring their function. Interestingly, group II proteins and Ra-KLP (PDBID: 2W8X) [24] are clustered into the same clade (Figure 5C). Ra-KLP, which is a salivary protein in Rhipicephalus appendiculatus (hard tick), can regulate host blood flow and innate immunity through modulating maxiK channels [24]. Ra-KLP is well superimposed with protein AAM93612 (group II) (RMSD: 1.2) (Figure 5B). The two proteins share strong similarities in the regions of the $3^{10}$ helix and the $\beta$-turn at the "base" of the structure (Figure 5B). Furthermore, structural elements ( $\mathrm{R}$ or $\mathrm{K}$ and a close hydrophobic residue [L, $\mathrm{Y}$, or $\mathrm{F}]$ ) associated with channel-modulating activity $[39,48]$ are present in the AAM93612 protein and most proteins in group II (Figure 5B and Additional File 5). These observations indicate that proteins in group II may modulate ion channels, such as maxiK channels. Some of the proteins in group II possess a $\mathrm{Q}$ instead of an R or $\mathrm{K}$ (Additional File 5), which means that these proteins cannot exhibit channel-modulating activity. Therefore, the possibility that group II may have multiple functions should not be excluded. 


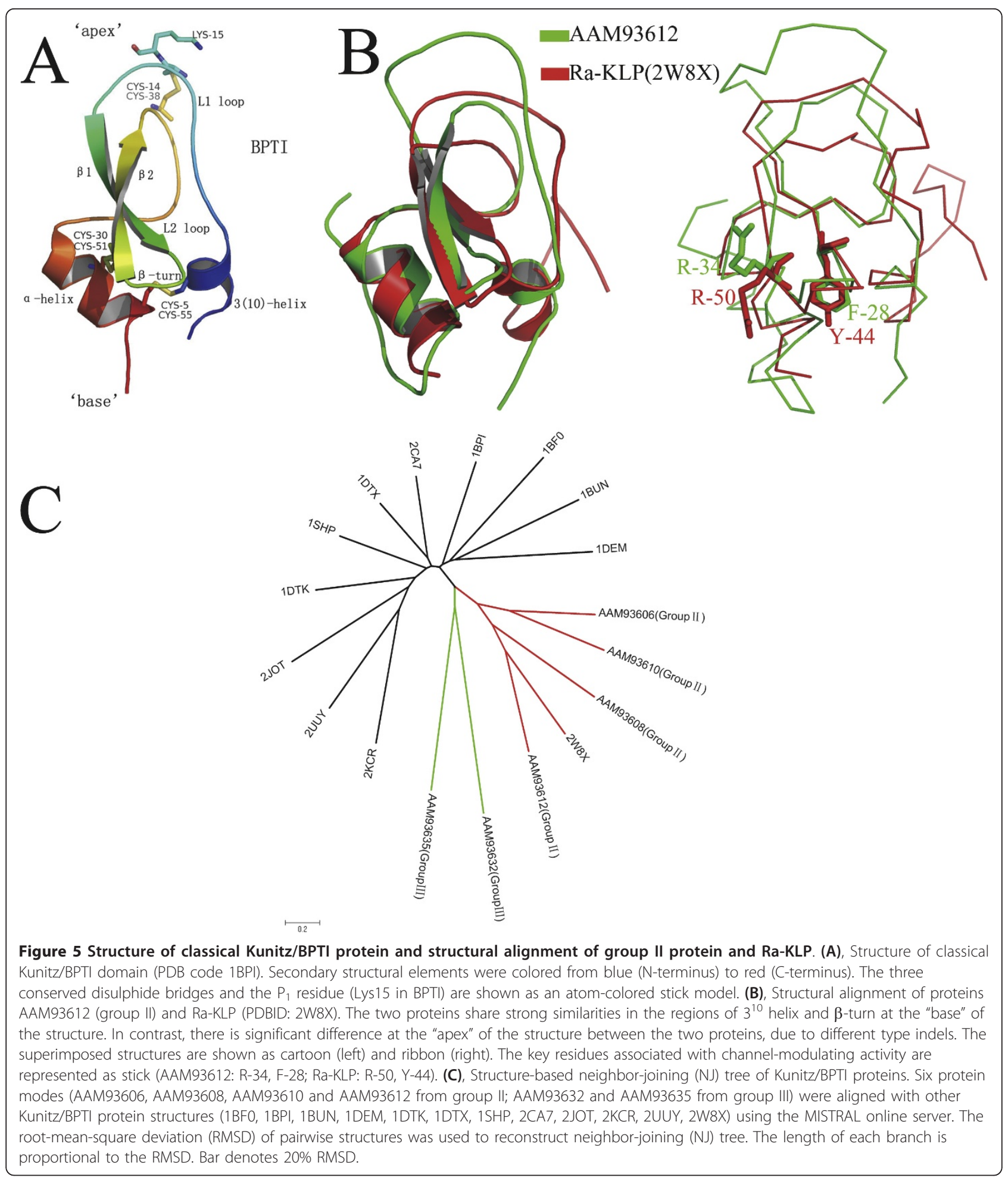

The expression patterns of genes in groups II and III exhibit stage-specificity during long-term blood feeding in Ixodes ticks

Hard ticks feed on blood for a few days to over one week, whereas soft ticks typically feed on blood for only minutes to hours [7]. Therefore, if genes exhibit significantly higher expression at 6-12 hours or later after host attachment, such genes may have a potentially important role in the long-term blood feeding of hard ticks. Here, we used R statistics [53] to analyze 


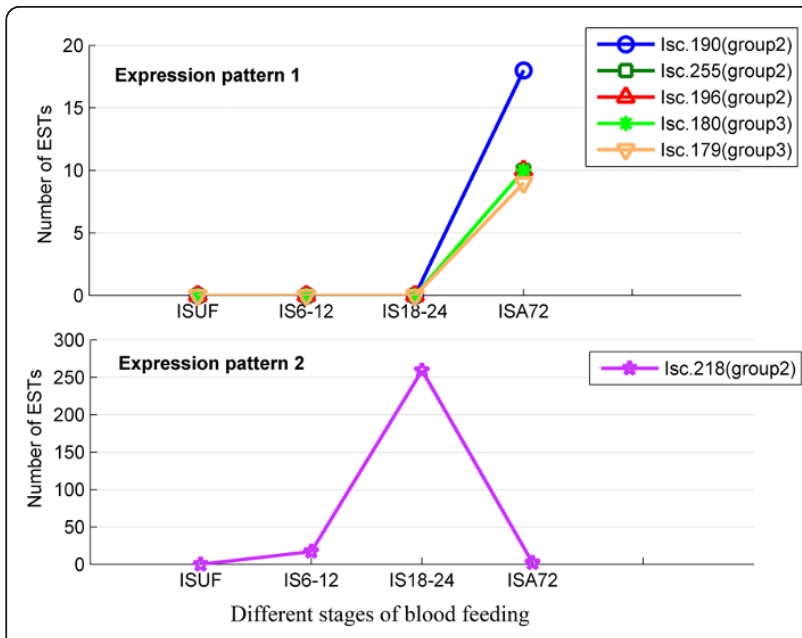

Figure 6 Expression patterns of six Kunitz/BPTI genes in Ixodes scapularis identified in our study. The number of ESTs for each gene is plotted against different stages of tick blood feeding (ISUF, IS6-12, S18-24 and ISA72). The details of the four stages are described in the Methods. Different genes are indicated by different colored makers. Expression of genes (Isc.190, Isc.255, Isc.196, Isc.180 and Isc.179) arises at 72 hours post host attachment (Expression pattern 1). However, expression of gene Isc.218 begins at 6-12 hours (17 ESTs), elevates strikingly at 18-24 hours (259 ESTs) and decreases rapidly at 72 hours (2 ESTs) post host attachment (Expression pattern 2).

four cDNA libraries to identify six differentially expressed genes $(R>=9$, Believability $>=99 \%$ ) belonging to the Kunitz/BPTI family during different stages of blood feeding in Ixodes scapularis (Table 1). All six genes were highly expressed at 6-12 hours or later after host attachment and two types of expression patterns were observed (Figure 6). The expression of genes Isc.190, Isc.255, Isc.196, Isc.180 and Isc.179 increases at 72 hours after host attachment (Figure 6Expression pattern 1). However, the expression of gene Isc.218 begins at 6-12 hours (17 ESTs), elevates strikingly at 18-24 hours (259 ESTs) and decreases rapidly at 72 hours (2 ESTs) after host attachment (Figure 6Expression pattern 2).

We further searched the EST database of Ixodes ricinus and found similar expression patterns for group II and III genes (Additional File 7). In both Ixodes scapularis and Ixodes ricinus, group II genes were expressed in the middle and late stages of long-term blood feeding, whereas group III genes were only expressed in the late stage of long-term blood feeding (Figure 6 and Additional File 7). The expression patterns of genes in groups II and III exhibit stage-specificity during longterm blood feeding. This suggests that these genes are functionally linked to long-term blood feeding in the Ixodes ticks.

\section{Positive selection drove the evolution of group II and III} genes

Positive selection is a major driving force in the expansion of gene families for particular functions [54]. To investigate whether positive selection drove the evolution of new functions in the Kunitz/BPTI family in Ixodes scapularis, site-specific models implemented in the CODEML program of PAML version 4.4c [55] were used to evaluate the selective pressure in each group. We found strong evidence (with p-value $<0.0001$ for M1a vs. M2a and M7 vs. M8) for positive selection imposed on both groups II and III, and we identified positively selected sites in each group (Figure 2B, 7 and Additional File 9 and 10). However, there no evidence for positive selection was found in group I (Figure 7 and Additional File 11).

Substitution saturation could bias the estimation of the $\mathrm{dN} / \mathrm{dS}$ ratios. In order to assess substitution saturation of three groups (group I, II and III) further, two methods (Steel's method [56] and Xia's method [57]) implemented in the software DAMBE [58] were used. Both methods show that group I has experienced substitution saturation, but groups II and III have experienced little substitution saturation (Additional file 12). Furthermore, previous research shows that saturation does not increase the rate of false positive predictions for the likelihood ratio test [59]. Therefore, the results of detection of positive selection within group II and III sequences remain relevant. Although Bayesian prediction of sites under positive selection is more sensitive to saturation level, the divergence levels estimated here do not pose a serious concern $[60,61]$. Therefore, we used detected sites under positive selection for further exploratory analyses.

Sequences used in the PAML analysis and the phylogenies with $\mathrm{dN}$ and $\mathrm{dS}$ on each branch have been provided in additional files (Additional File 13 and 14). There are more positively selected sites in group II than in group III (Figure 7, Additional File 9 and 10). To address whether the identified sites under positive selection relate to the molecular function of the Kunitz/BPTI family, the positively selected sites were mapped to three-dimensional structures modeled by I-TASSER online [62]. In group II, the positively selected sites are in the L1 and L2 loops at the "apex" and the $\alpha$-helix at the "base" of the structure (Figure 7B), which suggests that there was selection for rapid changes in this region. The rapid amino acid substitutions could lead to structural conformational changes in the $3^{10}$ helix and $\beta$ turn, which paves the way for the evolution of new functions, such as modulating ion channels. In group III, only three positively selected sites were identified; they are located at the L1 loop, L2 loop and $\beta$-turn 


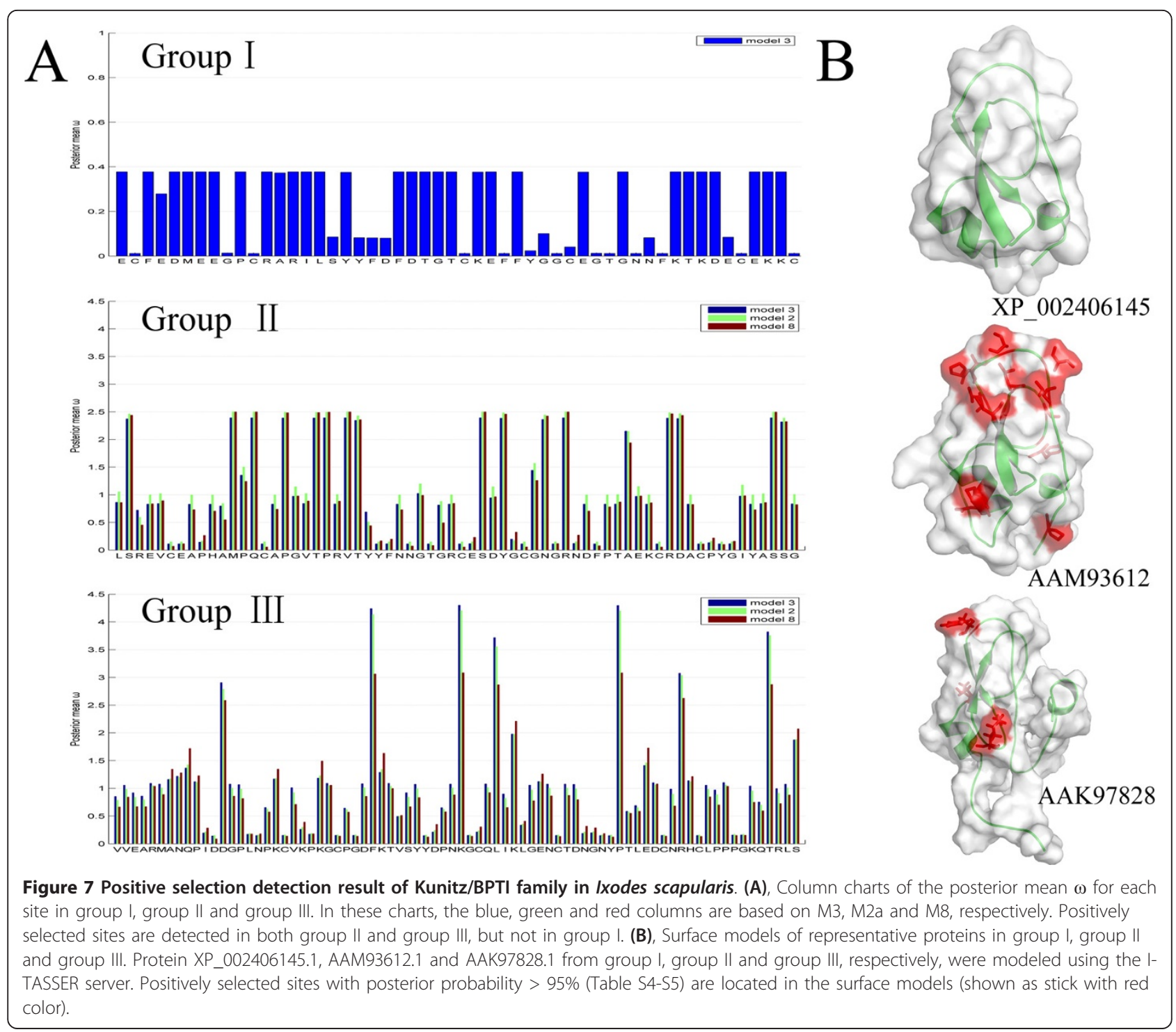

(Figure 7B), which suggests that these sites are involved in the function of group III proteins. In both group II and group III, positively selected sites are located in the functional regions. Therefore, rapid evolution in these two groups of Kunitz/BPTI proteins may have contributed to the evolution of new functions.

\section{Discussion}

The evolution scenario of Kunitz/BPTI family in ticks

Ticks are classified into several groups (Figure 8). Previous studies indicated that Ixodidae (hard ticks) evolved from bird-feeding soft ticks and whole genome duplication occurred once early in tick evolution $[15,63,64]$. Based on our results combined with those of previous studies, the evolution of the Kunitz/BPTI family in ticks is proposed as follows (Figure 8A). The common ancestor of ticks carried Kunitz/BPTI genes orthologous to those in group I, because group I genes are present in both soft and hard ticks. Hard and soft ticks diverged between 120 and 92 MYA [26], and whole genome duplication occurred in this divergence. Many multidomain Kunitz/BPTI proteins were created in hard ticks after the split between hard and soft ticks. Three-, four-, five- and seven-KU proteins are only present in hard ticks. After the split of Prostriata and Metastriata, certain indels developed in group I genes followed by multiple gene duplications gave rise to groups II and III in Prostriata. By contrast, other types of indels developed in group I genes in Metastriata. The group II and III type indels lead to structural variations in the Kunitz/ BPTI proteins (Figure 8B). For example, the L1 loop in group II turns into a longer and distorted loop, and the $3^{10}$ helix is not present in group III. These variations may result in evolutionary novelty by changing the 


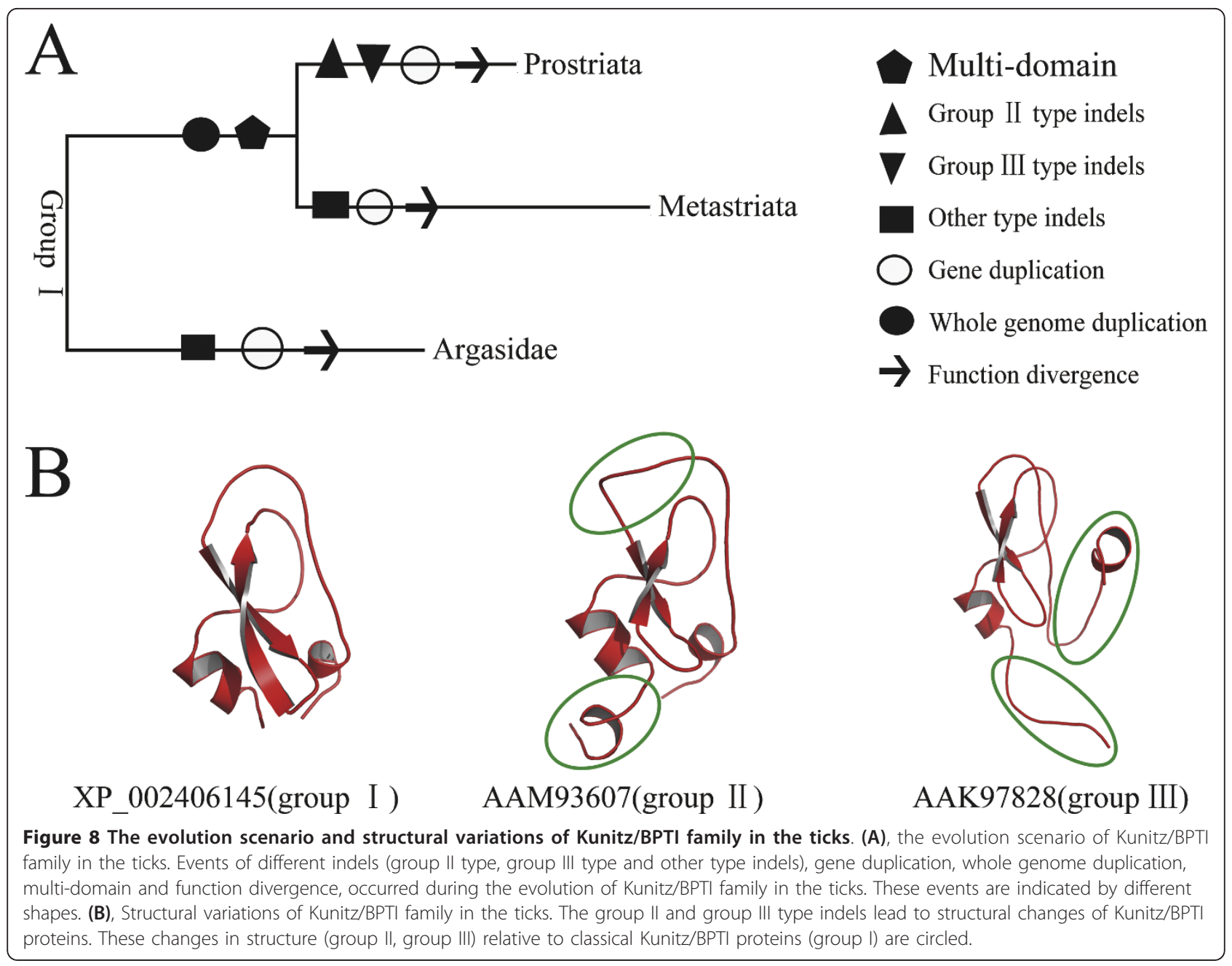

structure and therefore the function of the protein. Then, positive selection drove the divergence of genes in groups II and III in Prostriata, as described above. And structural alignment and functional site analysis suggests that genes in group II evolved a new function of modulating ion channels.

Ra-KLP, which is a salivary protein in Rhipicephalus appendiculatus (Metastriata), lost the function of inhibiting serine proteases but gained the function of modulating maxiK channels [24]. Both Ra-KLP and group II proteins are members of the Kunitz/BPTI family, but they resulted from different types of indels and formed different cysteine patterns after the split between Prostriata and Metastriata (Figure 5B, 8A and Additional File 15). However, the two proteins share strong similarities in their $3^{10}$ helices and $\beta$-turns at the "base" of the structures, and both have structural elements associated with channel-modulating activity (Figure $5 \mathrm{~B}$ and Additional File 15). This is an example of the phenomenon of convergent evolution. Kunitz/BPTI proteins in the two branches of hard ticks (Prostriata and Metastriata) acquired similar structures and functions despite having different indels and cysteine patterns (Figure 5B and Additional File 15).

This evolutionary scenario is rare in other species, such as snakes, spiders, cone snails and sea anemones, in which positive selection was detected in the evolution of the Kunitz/BPTI family despite the absence of indels [24,35-39].

\section{The profound role of Kunitz/BPTI family in long-term blood feeding}

To successfully feed to repletion, ticks must circumvent host hemostatic, inflammatory and immune responses [7-9]. Hemostatic responses rely on the triad of blood coagulation, platelet aggregation, and vasoconstriction [7]. In our study, group I proteins are serine protease inhibitors that function as anti-hemostatic factors by inhibiting kallikrein and the coagulation factors FXIIa, FXIa and FXa (Figure 3). Group II proteins may have evolved a new function in regulating host blood flow and innate immunity through modulating maxiK 
channels. Hard ticks imbibe large volumes of blood (approximately two thirds of their body volume) and expand enormously in the late stages of long-term blood feeding (named the rapid engorgement stage) $[65,66]$. Regulating host blood flow can enhance blood-feeding efficiency in the late stages of long-term blood feeding, which is beneficial for the rapid engorgement of hard ticks. Therefore, this function may play a profound role in the long-term blood feeding of hard ticks. Gene expression analysis reveals that the expression of genes in groups II and III is significantly higher in the late stages of long-term blood, which further suggests that these genes are functionally linked to long-term blood feeding.

\section{Different blood-feeding strategies may result from different sizes of the gene family in soft and hard ticks} Hard and soft ticks display different blood-feeding strategies: soft ticks typically feed for less than one hour, whereas hard ticks feed for prolonged periods of time, varying from a few days to over one week [7]. The reasons for this difference are not fully clarified $[1,7,12]$. Previous studies indicated that both hard and soft ticks possess a similar number of gene families, but they have different numbers of gene family members [12]. Multiple gene duplications occurred in hard ticks after whole genome duplication, resulting in a large number of gene family members. Compared with hard ticks, soft ticks possess fewer members in each family [12]. Therefore, different blood-feeding strategies may result from different sizes of the gene family in soft and hard ticks. In our study, there are a large number of Kunitz/BPTI genes, especially in the lineage-specific expanded genes (group II and III) in hard ticks. Group II and III proteins are functionally linked to long-term blood feeding. Furthermore, multi-domain proteins (three-, four-, fiveand seven-KU) are only present in hard ticks. In contrast, the few members of the Kunitz/BPTI family and the absence of groups II and III and multi-domain proteins in soft ticks may partly explain why soft ticks cannot feed on blood for longer periods.

\section{The six genes with highly dynamic expression identified in our study may be candidate target genes for tick control}

Tick control is important because ticks are vectors of a variety of bacterial and protozoan diseases, and ticks have the ability to transmit pathogens to vertebrates $[5,6]$. The development of vaccines directed against tick proteins may reduce tick infestations and the transmission of tick-borne pathogens. However, the limiting step in tick vaccine development is the identification of tick protective antigens [67-69]. Here, we propose that proteins encoded by the six genes (Table 1 ) identified in our study may be candidate protective antigens. This proposal is based on the following findings. First, the expression of these six genes increases significantly at 612 hours or later after host attachment. Additionally, these genes belong to groups II and III, which are subject to positive selection. The patterns of expression and evolution strongly suggest an important role for these genes in the process of long-term blood feeding. Second, safety issues may arise when protein ortholog sequences are used for vaccination because they can potentially induce autoimmune responses that are damaging to the host $[68,70]$. Fortunately, these group II and III genes are lineage specific and only present in Ixodes ticks. Although this feature makes these genes safe for vaccine development, it limits the application to only Ixodes ticks. Third, the Salp10 (AAK97828) protein in group III had been previously identified as a salivary gland antigen that elicited antibodies in the host [71]. This provides direct experimental evidence to support our proposal. Taken together, these six genes may be promising target genes for tick control. Additional experimental studies are needed to assess the validity of these genes for vaccine development.

\section{Conclusions}

Our study reveals the great differences between soft and hard ticks in the Kunitz/BPTI family. Compared with hard ticks, soft ticks do not possess group II and III proteins and multi-domain proteins (three-, four-, five- and seven-KU). Many multi-domain Kunitz/BPTI proteins were created in hard ticks using the group I domain as a module after the split between hard and soft ticks. Groups II and III, which exhibit significantly higher expression during long-term blood feeding, are only present and expanded in the genus Ixodes. In Ixodes, positive selection drove the expansion of the Kunitz/BPTI family and the evolution of new functions in group II such as ion channel-modulating ability. The two groups may play a profound role in the long-term blood feeding of hard ticks by enhancing blood-feeding efficiency in the late stages of long-term blood feeding, which is beneficial for the rapid engorgement of hard ticks. Therefore, our results suggest that the differences in the Kunitz/BPTI family between soft and hard ticks may be linked to the evolution of long-term blood feeding in hard ticks. Finally, we propose that the six genes (Isc.218, Isc.190, Isc.255, Isc.196, Isc.180 and Isc.179) identified in our study may be candidate target genes for tick control.

\section{Methods}

\section{Sequence retrieval and characteristics identification}

The search strategy used here is described as follow (Additional File 1). First, BPTI (a typical member of the 
Kunitz/BPTI family, UniProtKB AC: P00974) was used as a query sequence to search for Kunitz/BPTI protein sequences from all ticks against the NR database using BLASTP at the NCBI website under the default parameters $[72,73]$. Second, all hit sequences were filtered for the existence of a Kunitz/BPTI domain, which was determined by searching against the Pfam database under the default parameters [74]. To confirm that Kunitz/BPTI proteins were completely excavated, these putative Kunitz/BPTI proteins identified in this round of BLASTP search were used as a query to perform new rounds of BLASTP until no new hits appeared. Finally, a total of three rounds of BLASTP searches were performed, and there were 291, 368, and 368 sequences retrieved in the first, second and third rounds of the BLASTP search, respectively. Additionally, we used PSIBLAST to search for Kunitz/BPTI proteins. These two search strategies have similar performance (Additional File 16). All sequences identified as Kunitz/BPTI proteins were submitted to the SignalP 4.0 server [30] for signal peptide prediction. Gene structures of the Kunitz/ BPTI family were determined using the gene structure display server http://gsds.cbi.pku.edu.cn/index.php[75].

\section{Phylogenetic analysis of the Kunitz/BPTI family}

To facilitate analysis of the Kunitz/BPTI family in ticks, Kunitz/BPTI proteins were classified into the following categories based their domain architectures: singledomain Kunitz/BPTI proteins present only in Ixodes Scapularis (ixosc_1domain), single-domain Kunitz/BPTI proteins present in all ticks (all_1domain), two-domain Kunitz/BPTI proteins present in all ticks (all_2domain), three-domain Kunitz/BPTI proteins present in all ticks (all_3domain), four-domain Kunitz/BPTI proteins present in all ticks (all_4domain), five-domain Kunitz/BPTI proteins present in all ticks (all_5domain), seven-domain Kunitz/BPTI proteins present in all ticks (all_7domain), and the combination of single-domain and multidomain Kunitz/BPTI proteins present only in Ixodes scapularis (ixosc_mix). Proteins in each category were aligned using ClustalW 2.0 [76] with fine adjustment by hand with reference to the cysteine residue position. Because the Kunitz domains are subjected to indels, ClustalW may not have treated these indels correctly. Therefore, the program Prank $[77,78]$ was used to infer the indels and confirm the alignments obtained from ClustalW. Based on the alignment above, amino acid substitution models of protein evolution were determined by ProtTest 2.4 [79]. Phylogeny inference was performed using the neighbor-joining (NJ), Bayesian inference (MB) and maximum likelihood (ML) methods. MEGA 4.0 [80], MrBayes 3.1.2 [81] and Tree Finder [82] were used for reconstructing the NJ, MB and ML trees, respectively. All sets of parameters used for sequence alignment, model testing and phylogenetic analysis were listed (Additional File 17).

\section{Evolutionary analysis of the Kunitz/BPTI family}

Three groups (groups I, II and III) from single-domain Kunitz/BPTI proteins in Ixodes scapularis were tested to determine whether they were subjected to selection. Tests of selection (nonsynonymous-to-synonymous rate ratio, $\omega=\mathrm{dN} / \mathrm{dS}$ ) were accomplished by using codonbased substitution models implemented in the CODEML program of PAML version 4.4c [55]. Codon alignments of nucleotide sequences (Additional File 13) for three groups were constructed from the PAL2NAL server [83] based corresponding protein sequence alignments. The tree topology used for the three groups was based on the NJ trees in Additional File 14. The presence of a positively selected rate class was detected using the likelihood ratio tests (LRTs, i.e., by comparing the likelihood of a neutral model with that of a selection model). For detailed assumptions and descriptions of each model see papers $[55,84]$. The Model 0 (M0) assumes one $\omega$ for all sites. Three pairs of models make three LRTs by comparing M0 (one ratio) against M3 (discrete), M1a (nearly neutral model) against M2a (positive selection model) and M7 ( $\beta$ distribution model) against M8 ( $\beta \& \omega$ model). To test for significance, the LRT statistic $2 \Delta \mathrm{L}$ (twice the log likelihood difference) was compared against the chi-square distribution (with $\mathrm{df}=2$ with critical values of 9.21 for M1a vs. M2a and $\mathrm{M} 7$ vs. M8, but with $\mathrm{df}=4$ with critical values 13.28 for M0 vs. M3) at the $1 \%$ significance level. The Bayes Empirical Bayes (BEB) approach was used to calculate the posterior probability that each site was from a particular site class, and sites with high posterior probabilities coming from the class with $\omega>1$ (with $\mathrm{p}>0.95$ ) were inferred to be under positive selection. All analyses were run twice using different initial $\omega$ values to ensure convergence. Substitution saturation could bias the estimation of the $\mathrm{dN} / \mathrm{dS}$ ratios. In order to assess substitution saturation of three groups (group I, II and III), two methods (Steel's method [56] and Xia's method [57]) implemented in the software DAMBE [58] were used.

\section{Protein modeling, structural alignment and locating positively selected sites}

Nine proteins (XP_002406145 from group I; AAM93606, AAM93607, AAM93608, AAM93610 and AAM93612 from group II; AAM93632, AAM93635 and AAK97828 from group III) were modeled using the ITASSER server $[62,85]$. All model structures predicted here had been deposited in Protein Model DataBase [86] under ID numbers PM0077184-PM0077186 and PM0077307-PM0077312. Structural alignment was performed using the MISTRAL online server [87]. PyMOL 
[88] was used to visualize the structure and locate the positively selected sites.

\section{Comparison of the expression levels in different stages of blood feeding}

To estimate the differential expression of Ixodes scapularis genes in the different stages of blood feeding, four cDNA libraries from Ixodes scapularis adult female ticks, which were constructed following the same protocol described in a previous paper [15], were downloaded from the NCBI website for in silico analysis. The four cDNA libraries are as follows: 1 ) adult females, unfed (ISUF); 2) adult females, 6-12 hours after host attachment (IS6-12); 3) adult females, 18-24 hours after host attachment (IS18-24); and 4) adult females, 3-4 days after host attachment (ISA72). Details of the libraries were shown (Additional File 18). Then these four cDNA libraries were analyzed using $R$ statistics [53], which is a method based on the comparison of the abundance of ESTs contributing to each contig in the different cDNA libraries. This method allows for the comparison of gene expression in any number of libraries, and it can identify differentially expressed genes as those with a high R-value. A Perl program was written to conduct this analysis (Additional File 13). The expression of Ixodes ricinus genes in the different stages of blood feeding was also analyzed. Because the cDNA libraries and Unigene database for Ixodes ricinus are unavailable, $\mathrm{R}$ statistics is not applicable in this case. We searched for Kunitz/BPTI sequences against the EST database of Ixodes ricinus in NCBI by TBLASTN. Based the annotation of the ESTs, we obtained gene expression information for the Kunitz/BPTI family in the different stages of blood feeding.

\section{Additional material}

Additional file 1: Figure S1. Two strategies for database searches of ticks Kunitz/BPTI proteins from NR database in NCBI.

Additional file 2: Table S1. Details of removed protein sequences.

Additional file 3: Table S2. Detailed information for all 303 sequences.

Additional file 4: Figure S2. Neighbor-joining (NJ) and maximum likelihood (ML) tree of single-domain Kunitz/BPTI proteins in Ixodes scapularis.

Additional file 5: Figure S3. Alignment of single-domain Kunitz/BPTI proteins in Ixodes scapularis.

Additional file 6: Figure S4. Neighbor-joining (NJ) and maximum likelihood (ML) tree of all single-domain Kunitz/BPTI proteins in ticks.

Additional file 7: Figure S5. Alignment of group II and group III Kunitz/ BPTI protein sequences translated from ESTs in the lxodes ricinus.

Additional file 8: Figure S6. Bayesian inference (MB) tree of single and multi-domain Kunitz/BPTI proteins in Ixodes scapularis.

Additional file 9: Table S3. Results of selection test for group II. Additional file 10: Table S4. Results of selection test for group III. Additional file 11: Table S5. Results of selection test for group I.
Additional file 12: S1. Results for assessing substitution saturation of the three groups.

Additional file 13: S2. Codon alignments of nucleotide sequences for three groups and script of the Perl program used in our study.

Additional file 14: Figure S7. Phylogeny of group I, II and III and for PAML analyze with dN and dS on each branch.

Additional file 15: Figure S8. Alignment of Ra-KLP related proteins in Metastriata ticks and group II proteins in Prostriata ticks.

Additional file 16: Table S6. Two search strategies have similar performance.

Additional file 17: Table S7. All sets of parameters used for sequence and phylogenetic analysis.

Additional file 18: Table S8. Details of the four CDNA libraries used for the expression analysis.

\section{Acknowledgements}

We thank our colleagues, Drs. Gong-Hua Li, Zi-Zhang Sheng and DongQiang Cheng and Yu-Qi Zhao for helpful comments on the manuscript. We also thank two anonymous reviewers for valuable comments. This work was supported by the National Basic Research Program of China (Grant No. 2007CB815705, 2009CB941300), the National Natural Science Foundation of China (Grant No.30623007) and Chinese Academy of Sciences (Grant No.2007211311091) for JFH.

\section{Author details}

${ }^{1}$ School of Life Sciences, University of Science and Technology of China, Hefei, Anhui 230027, P.R. China. ${ }^{2}$ State Key Laboratory of Genetic Resources and Evolution, Kunming Institute of Zoology, Chinese Academy of Sciences, 32, Eastern Jiaochang Road, Kunming, Yunnan 650223, P.R. China.

\section{Authors' contributions}

SXD and ADZ carried out the data mining, the sequence alignments, bioinformatic analysis and wrote the manuscript. JFH designed and coordinated the study and participated in writing the manuscript. All authors read and approved the final manuscript.

Received: 26 July 2011 Accepted: 14 January 2012

Published: 14 January 2012

\section{References}

1. Nava S, Guglielmone AA, Mangold AJ: An overview of systematics and evolution of ticks. Front Biosci 2009, 14:2857-2877.

2. Black WCt, Piesman J: Phylogeny of hard- and soft-tick taxa (Acari: Ixodida) based on mitochondrial $16 \mathrm{~S}$ rDNA sequences. Proc Natl Acad Sci USA 1994, 91(21):10034-10038.

3. Nuttall PA, Paesen GC, Lawrie $\mathrm{CH}$, Wang H: Vector-host interactions in disease transmission. J Mol Microbiol Biotechnol 2000, 2(4):381-386.

4. Ullmann AJ, Lima CM, Guerrero FD, Piesman J, Black WCt: Genome size and organization in the blacklegged tick, Ixodes scapularis and the Southern cattle tick, Boophilus microplus. Insect Mol Biol 2005, 14(2):217-222.

5. Belongia EA: Epidemiology and impact of coinfections acquired from Ixodes ticks. Vector Borne Zoonotic Dis 2002, 2(4):265-273.

6. Krause PJ: Babesiosis. Med Clin North Am 2002, 86(2):361-373.

7. Francischetti IM, Sa-Nunes A, Mans BJ, Santos IM, Ribeiro JM: The role of saliva in tick feeding. Front Biosci 2009, 14:2051-2088.

8. Corral-Rodriguez MA, Macedo-Ribeiro S, Barbosa Pereira PJ, Fuentes-Prior P: Tick-derived Kunitz-type inhibitors as antihemostatic factors. Insect Biochem Mol Biol 2009, 39(9):579-595.

9. Andersen JF: Structure and mechanism in salivary proteins from bloodfeeding arthropods. Toxicon 2009.

10. Ribeiro JM, Francischetti IM: Role of arthropod saliva in blood feeding: sialome and post-sialome perspectives. Annu Rev Entomol 2003, 48:73-88.

11. Mans BJ, Andersen JF, Schwan TG, Ribeiro JM: Characterization of antihemostatic factors in the argasid, Argas monolakensis: implications for 
the evolution of blood-feeding in the soft tick family. Insect Biochem $\mathrm{Mol}$ Biol 2008, 38(1):22-41.

12. Mans BJ, Andersen JF, Francischetti IM, Valenzuela JG, Schwan TG, Pham VM, Garfield MK, Hammer CH, Ribeiro JM: Comparative sialomics between hard and soft ticks: implications for the evolution of bloodfeeding behavior. Insect Biochem Mol Biol 2008, 38(1):42-58.

13. Francischetti IM, Meng Z, Mans BJ, Gudderra N, Hall M, Veenstra TD, Pham VM, Kotsyfakis M, Ribeiro JM: An insight into the salivary transcriptome and proteome of the soft tick and vector of epizootic bovine abortion, Ornithodoros coriaceus. J Proteomics 2008, 71(5):493-512.

14. Francischetti IM, Mans BJ, Meng Z, Gudderra N, Veenstra TD, Pham VM, Ribeiro JM: An insight into the sialome of the soft tick, Ornithodorus parkeri. Insect Biochem Mol Biol 2008, 38(1):1-21.

15. Ribeiro JM, Alarcon-Chaidez F, Francischetti IM, Mans BJ, Mather TN, Valenzuela JG, Wikel SK: An annotated catalog of salivary gland transcripts from Ixodes scapularis ticks. Insect Biochem Mol Biol 2006, 36(2):111-129.

16. Anatriello E, Ribeiro JMC, de Miranda-Santos IKF, Brandao LG, Anderson JM, Valenzuela JG, Maruyama SR, Silva JS, Ferreira BR: An insight into the sialotranscriptome of the brown dog tick, Rhipicephalus sanguineus. BMC Genomics 2010, 11.

17. Francischetti IM, My Pham V, Mans BJ, Andersen JF, Mather TN, Lane RS, Ribeiro JM: The transcriptome of the salivary glands of the female western black-legged tick Ixodes pacificus (Acari: Ixodidae). Insect Biochem Mol Biol 2005, 35(10):1142-1161.

18. Valenzuela JG, Francischetti IM, Pham VM, Garfield MK, Mather TN, Ribeiro JM: Exploring the sialome of the tick Ixodes scapularis. J Exp Biol 2002, 205(Pt 18):2843-2864.

19. Ribeiro JM, Anderson JM, Manoukis NC, Meng Z, Francischetti IM: A further insight into the sialome of the tropical bont tick, Amblyomma variegatum. BMC Genomics 2011, 12:136.

20. Chmelař RJ: Transcriptomic and functional analysis of salivary proteins from the tick. PhD Thesis 2010.

21. Andrade BB, Teixeira CR, Barral A, Barral-Netto M: Haematophagous arthropod saliva and host defense system: a tale of tear and blood. An Acad Bras Cienc 2005, 77(4):665-693.

22. Chen C, Hsu CH, Su NY, Lin YC, Chiou SH, Wu SH: Solution structure of a Kunitz-type chymotrypsin inhibitor isolated from the elapid snake Bungarus fasciatus. J Biol Chem 2001, 276(48):45079-45087.

23. Schmidt AE, Chand HS, Cascio D, Kisiel W, Bajaj SP: Crystal structure of Kunitz domain 1 (KD1) of tissue factor pathway inhibitor-2 in complex with trypsin-Implications for KD1 specificity of inhibition. Journal of Biological Chemistry 2005, 280(30):27832-27838.

24. Paesen GC, Siebold C, Dallas ML, Peers C, Harlos K, Nuttall PA, Nunn MA, Stuart DI, Esnouf RM: An ion-channel modulator from the saliva of the brown ear tick has a highly modified Kunitz/BPTI structure. J Mol Biol 2009, 389(4):734-747.

25. Miyoshi T, Tsuji N, Islam MK, Alim MA, Hatta T, Yamaji K, Anisuzzaman, Fujisaki K: A Kunitz-type proteinase inhibitor from the midgut of the ixodid tick, Haemaphysalis longicornis, and its endogenous target serine proteinase. Mol Biochem Parasitol 2010, 170(2):112-115.

26. Mans BJ, Louw Al, Neitz AW: Evolution of hematophagy in ticks: common origins for blood coagulation and platelet aggregation inhibitors from soft ticks of the genus Ornithodoros. Mol Biol Evol 2002, 19(10):1695-1705.

27. Islam MK, Tsuji N, Miyoshi T, Alim MA, Huang XH, Hatta T, Fujisaki K: The Kunitz-Like Modulatory Protein Haemangin Is Vital for Hard Tick BloodFeeding Success. Plos Pathogens 2009, 5(7).

28. Hill CA, Wikel SK: The Ixodes scapularis Genome Project: an opportunity for advancing tick research. Trends Parasitol 2005, 21(4):151-153.

29. Mulenga A, Khumthong R, Chalaire KC: Ixodes scapularis tick serine proteinase inhibitor (serpin) gene family; annotation and transcriptional analysis. BMC Genomics 2009, 10:217.

30. Petersen TN, Brunak S, von Heijne G, Nielsen H: SignalP 4.0: discriminating signal peptides from transmembrane regions. Nat Methods 2011, 8(10):785-786.

31. Gao X, Shi L, Zhou Y, Cao J, Zhang H, Zhou J: Characterization of the anticoagulant protein Rhipilin-1 from the Rhipicephalus haemaphysaloides tick. J Insect Physiol 2011, 57(2):339-343.

32. Ceraul SM, Chung A, Sears KT, Popov VL, Beier-Sexton M, Rahman MS, Azad AF: A Kunitz protease inhibitor from Dermacentor variabilis, a vector for spotted fever group rickettsiae, limits Rickettsia montanensis invasion. Infect Immun 2011, 79(1):321-329.

33. Islam MK, Tsuji N, Miyoshi T, Alim MA, Huang X, Hatta T, Fujisaki K: The Kunitz-like modulatory protein haemangin is vital for hard tick bloodfeeding success. PLoS Pathog 2009, 5(7):e1000497.

34. Lima CA, Torquato RJ, Sasaki SD, Justo GZ, Tanaka AS: Biochemical characterization of a Kunitz type inhibitor similar to dendrotoxins produced by Rhipicephalus (Boophilus) microplus (Acari: Ixodidae) hemocytes. Vet Parasitol 2010, 167(2-4):279-287.

35. Schweitz H, Bruhn T, Guillemare E, Moinier D, Lancelin JM, Beress L, Lazdunski M: Kalicludines and Kaliseptine-2 Different Classes of SeaAnemone Toxins for Voltage-Sensitive K+ Channels. Journal of Biological Chemistry 1995, 270(42):25121-25126.

36. Yuan CH, He QY, Peng K, Diao JB, Jiang LP, Tang X, Liang SP: Discovery of a distinct superfamily of Kunitz-type toxin (KTT) from tarantulas. PLOS One 2008, 3(10):e3414

37. Bayrhuber M, Vijayan V, Ferber M, Graf R, Korukottu J, Imperial J, Garrett JE, Olivera BM, Terlau H, Zweckstetter M, et al: Conkunitzin-S1 is the first member of a new Kunitz-type neurotoxin family-Structural and functional characterization. Journal of Biological Chemistry 2005, 280(25):23766-23770.

38. Zupunski V, Kordis D, Gubensek F: Adaptive evolution in the snake venom Kunitz/BPTI protein family. FEBS Lett 2003, 547(1-3):131-136.

39. Harvey AL: Twenty years of dendrotoxins. Toxicon 2001, 39(1):15-26.

40. Macedo-Ribeiro S, Almeida C, Calisto BM, Friedrich T, Mentele R, Sturzebecher J, Fuentes-Prior P, Pereira PJ: Isolation, cloning and structural characterisation of boophilin, a multifunctional Kunitz-type proteinase inhibitor from the cattle tick. PLoS One 2008, 3(2):e1624.

41. Navaneetham D, Jin L, Pandey P, Strickler JE, Babine RE, Abdel-Meguid SS, Walsh PN: Structural and mutational analyses of the molecular interactions between the catalytic domain of factor Xla and the Kunitz protease inhibitor domain of protease nexin 2. J Biol Chem 2005, 280(43):36165-36175

42. Chand HS, Schmidt AE, Bajaj SP, Kisiel W: Structure-function analysis of the reactive site in the first Kunitz-type domain of human tissue factor pathway inhibitor-2 (vol 279, pg 17500, 2004). Journal of Biological Chemistry 2004, 279(23):24906-24906.

43. Denda K, Shimomura T, Kawaguchi T, Miyazawa K, Kitamura N: Functional characterization of Kunitz domains in hepatocyte growth factor activator inhibitor type 1. J Biol Chem 2002, 277(16):14053-14059.

44. Schmidt AE, Chand HS, Cascio D, Kisiel W, Bajaj SP: Crystal structure of Kunitz domain 1 (KD1) of tissue factor pathway inhibitor-2 in complex with trypsin. Implications for KD1 specificity of inhibition. J Biol Chem 2005, 280(30):27832-27838.

45. St Pierre L, Earl ST, Filippovich I, Sorokina N, Masci PP, De Jersey J, Lavin MF: Common evolution of waprin and kunitz-like toxin families in Australian venomous snakes. Cell Mol Life Sci 2008, 65(24):4039-4054.

46. Otlewski J, Jaskolski M, Buczek O, Cierpicki T, Czapinska H, Krowarsch D, Smalas AO, Stachowiak D, Szpineta A, Dadlez M: Structure-function relationship of serine protease-protein inhibitor interaction. Acta Biochim Pol 2001, 48(2):419-428.

47. Parkin S, Rupp B, Hope H: Structure of bovine pancreatic trypsin inhibitor at $125 \mathrm{~K}$ : Definition of carboxyl-terminal residues Gly57 and Ala58. Acta Crystallogr D 1996, 52:18-29.

48. Gasparini S, Danse JM, Lecoq A, Pinkasfeld S, Zinn-Justin S, Young LC, de Medeiros CC, Rowan EG, Harvey AL, Menez A: Delineation of the functional site of alpha-dendrotoxin. The functional topographies of dendrotoxins are different but share a conserved core with those of other Kv1 potassium channel-blocking toxins. J Biol Chem 1998, 273(39):25393-25403.

49. Katoh E, Nishio H, Inui T, Nishiuchi Y, Kimura T, Sakakibara S, Yamazaki T: Structural basis for the biological activity of dendrotoxin-I, a potent potassium channel blocker. Biopolymers 2000, 54(1):44-57.

50. Nishio H, Katoh E, Yamazaki T, Inui T, Nishiuchi Y, Kimura T: Structureactivity relationships of calcicludine and dendrotoxin-I, homologous peptides acting on different targets, calcium and potassium channels. Biochem Biophys Res Commun 1999, 262(2):319-321.

51. Gonzalez S, Flo M, Margenat M, Duran R, Gonzalez-Sapienza G, Grana M, Parkinson J, Maizels RM, Salinas G, Alvarez B, et al: A Family of Diverse Kunitz Inhibitors from Echinococcus granulosus Potentially Involved in Host-Parasite Cross-Talk. PLoS One 2009, 4(9). 
52. Decrem Y, Rath G, Blasioli V, Cauchie P, Robert S, Beaufays J, Frere JM, Feron O, Dogne JM, Dessy C, et al: Ir-CPI, a coagulation contact phase inhibitor from the tick Ixodes ricinus, inhibits thrombus formation without impairing hemostasis. J Exp Med 2009, 206(11):2381-2395.

53. Stekel DJ, Git Y, Falciani F: The comparison of gene expression from multiple cDNA libraries. Genome Res 2000, 10(12):2055-2061.

54. Wu DD, Wang GD, Irwin DM, Zhang YP: A profound role for the expansion of trypsin-like serine protease family in the evolution of hematophagy in mosquito. Mol Biol Evol 2009, 26(10):2333-2341.

55. Yang Z: PAML 4: phylogenetic analysis by maximum likelihood. Mol Biol Evol 2007, 24(8):1586-1591.

56. Steel MA, Lockhart PJ, Penny D: Confidence in Evolutionary Trees from Biological Sequence Data. Nature 1993, 364(6436):440-442.

57. Xia X, Xie Z, Salemi M, Chen L, Wang Y: An index of substitution saturation and its application. Mol Phylogenet Evol 2003, 26(1):1-7.

58. Xia X, Xie Z: DAMBE: Software package for data analysis in molecular biology and evolution. Journal of Heredity 2001, 92(4):371-373.

59. Anisimova M, Bielawski JP, Yang ZH: Accuracy and power of the likelihood ratio test in detecting adaptive molecular evolution. Molecular Biology and Evolution 2001, 18(8):1585-1592.

60. Anisimova M, Bielawski JP, Yang Z: Accuracy and power of bayes prediction of amino acid sites under positive selection. Mol Biol Evol 2002, 19(6):950-958

61. Yang Z, Wong WS, Nielsen R: Bayes empirical bayes inference of amino acid sites under positive selection. Mol Biol Evol 2005, 22(4):1107-1118.

62. Roy A, Kucukural A, Zhang Y: I-TASSER: a unified platform for automated protein structure and function prediction. Nat Protoc 2010, 5(4):725-738.

63. Geraci NS, Spencer Johnston J, Paul Robinson J, Wikel SK, Hill CA: Variation in genome size of argasid and ixodid ticks. Insect Biochem Mol Biol 2007, 37(5):399-408.

64. Mans BJ, Neitz AW: Adaptation of ticks to a blood-feeding environment: evolution from a functional perspective. Insect Biochem Mol Biol 2004, 34(1):1-17.

65. Franta Z, Frantova H, Konvickova J, Horn M, Sojka D, Mares M, Kopacek P. Dynamics of digestive proteolytic system during blood feeding of the hard tick Ixodes ricinus. Parasit Vectors 2010, 3:119.

66. Tsuda A, Mulenga A, Sugimoto C, Nakajima M, Ohashi K, Onuma M: cDNA cloning, characterization and vaccine effect analysis of Haemaphysalis longicornis tick saliva proteins. Vaccine 2001, 19(30):4287-4296.

67. Prudencio CR, Marra AOM, Cardoso R, Goulart LR: Recombinant peptides as new immunogens for the control of the bovine tick, Rhipicephalus (Boophilus) microplus. Veterinary Parasitology 2010, 172(1-2):122-131.

68. de la Fuente J, Manzano-Roman R, Naranjo V, Kocan KM, Zivkovic Z Blouin EF, Canales M, Almazan C, Galindo RC, Step DL, et al: Identification of protective antigens by RNA interference for control of the lone star tick, Amblyomma americanum. Vaccine 2010, 28(7):1786-1795.

69. Dryden MW: Flea and tick control in the 21st century: challenges and opportunities. Vet Dermatol 2009, 20(5-6):435-440.

70. Canales M, Naranjo V, Almazan C, Molina R, Tsuruta SA, Szabo MPJ, Manzano-Roman R, de la Lastra JMP, Kocan KM, Jimenez Ml, et al: Conservation and immunogenicity of the mosquito ortholog of the tickprotective antigen, subolesin. Parasitology Research 2009, 105(1):97-111.

71. Das S, Banerjee G, DePonte K, Marcantonio N, Kantor FS, Fikrig E: Salp25D, an Ixodes scapularis antioxidant, is 1 of 14 immunodominant antigens in engorged tick salivary glands. Journal of Infectious Diseases 2001, 184(8):1056-1064.

72. Altschul SF, Gish W, Miller W, Myers EW, Lipman DJ: Basic local alignment search tool. J Mol Biol 1990, 215(3):403-410.

73. Camacho C, Coulouris G, Avagyan V, Ma N, Papadopoulos J, Bealer K, Madden TL: BLAST+: architecture and applications. BMC Bioinformatics 2009, 10:421.

74. Finn RD, Mistry J, Tate J, Coggill P, Heger A, Pollington JE, Gavin OL, Gunasekaran P, Ceric G, Forslund K, et al: The Pfam protein families database. Nucleic Acids Research 2010, 38:D211-D222.

75. Guo AY, Zhu QH, Chen X, Luo JC: [GSDS: a gene structure display server]. Yi Chuan 2007, 29(8):1023-1026.

76. Larkin MA, Blackshields G, Brown NP, Chenna R, McGettigan PA, McWilliam H, Valentin F, Wallace IM, Wilm A, Lopez R, et al: Clustal W and Clustal X version 2.0. Bioinformatics 2007, 23(21):2947-2948.
77. Loytynoja A, Goldman N: webPRANK: a phylogeny-aware multiple sequence aligner with interactive alignment browser. BMC Bioinformatics 2010, 11:579.

78. Loytynoja A, Goldman N: An algorithm for progressive multiple alignment of sequences with insertions. Proc Natl Acad Sci USA 2005, 102(30):10557-10562.

79. Abascal F, Zardoya R, Posada D: ProtTest: selection of best-fit models of protein evolution. Bioinformatics 2005, 21(9):2104-2105.

80. Tamura K, Dudley J, Nei M, Kumar S: MEGA4: Molecular evolutionary genetics analysis (MEGA) software version 4.0. Molecular Biology and Evolution 2007, 24(8):1596-1599.

81. Ronquist F, Huelsenbeck JP: MrBayes 3: Bayesian phylogenetic inference under mixed models. Bioinformatics 2003, 19(12):1572-1574.

82. Jobb G, von Haeseler A, Strimmer K: TREEFINDER: a powerful graphical analysis environment for molecular phylogenetics. Bmc Evolutionary Biology 2004, 4.

83. Suyama M, Torrents D, Bork P: PAL2NAL: robust conversion of protein sequence alignments into the corresponding codon alignments. Nucleic Acids Res 2006, , 34 Web Server: W609-612.

84. Yang Z, Swanson WJ, Vacquier VD: Maximum-likelihood analysis of molecular adaptation in abalone sperm lysin reveals variable selective pressures among lineages and sites. Mol Biol Evol 2000, 17(10):1446-1455.

85. Zhang Y: I-TASSER server for protein 3D structure prediction. BMC Bioinformatics 2008, 9:40.

86. Castrignano T, De Meo PD, Cozzetto D, Talamo IG, Tramontano A: The PMDB Protein Model Database. Nucleic Acids Res 2006, , 34 Database: D306-309.

87. Micheletti C, Orland H: MISTRAL: a tool for energy-based multiple structural alignment of proteins. Bioinformatics 2009, 25(20):2663-2669.

88. DeLano WL: The PyMOL Molecular Graphics System. DeLano Scientific San Carlos, CA, USA; 2002.

doi:10.1186/1471-2148-12-4

Cite this article as: Dai et al:: Evolution, expansion and expression of the Kunitz/BPTI gene family associated with long-term blood feeding in Ixodes Scapularis. BMC Evolutionary Biology 2012 12:4.

\section{Submit your next manuscript to BioMed Central and take full advantage of:}

- Convenient online submission

- Thorough peer review

- No space constraints or color figure charges

- Immediate publication on acceptance

- Inclusion in PubMed, CAS, Scopus and Google Scholar

- Research which is freely available for redistribution

Submit your manuscript at www.biomedcentral.com/submit 